\title{
New Frontiers Mission Concept Study to Explore Oort Cloud Comets
}

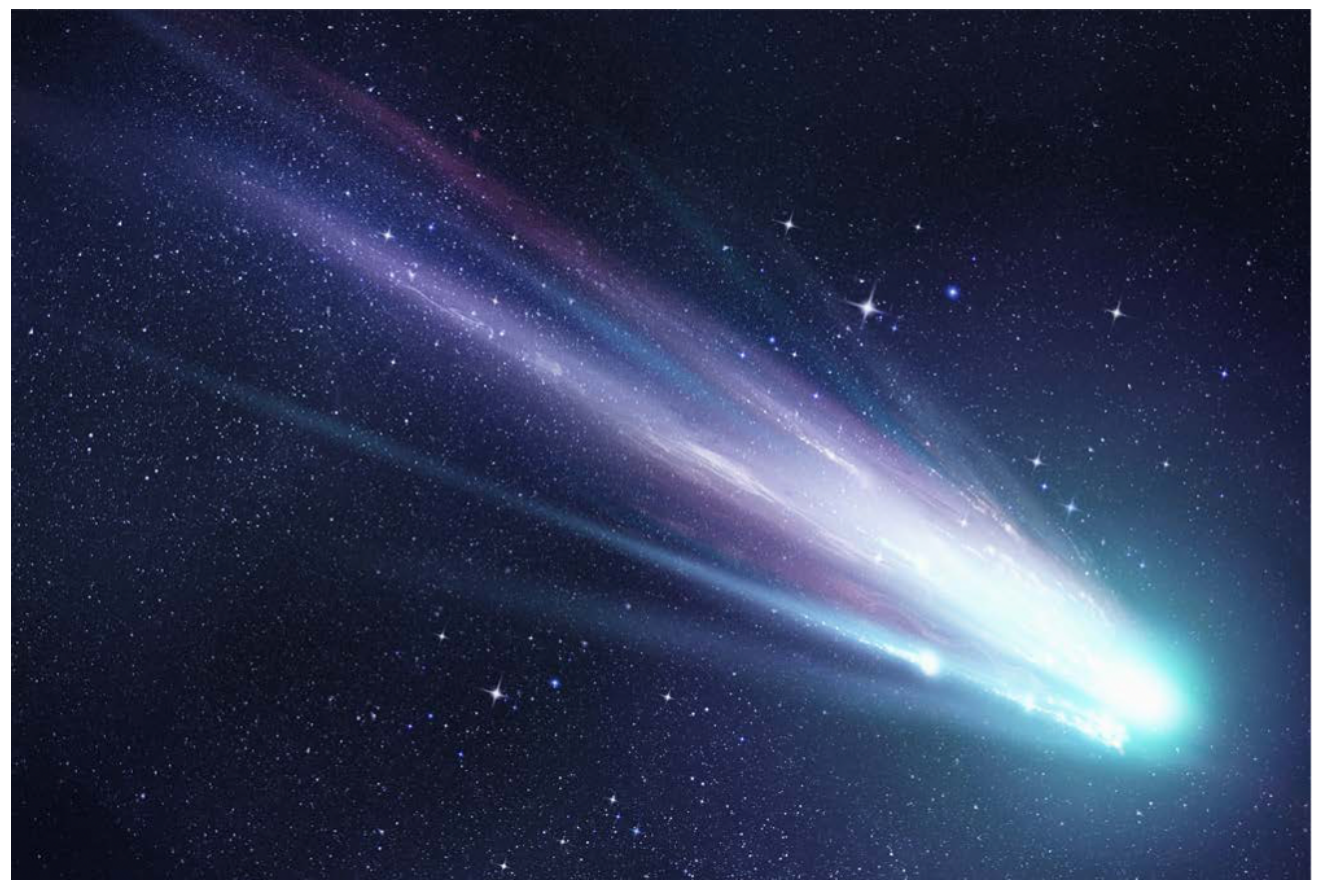

\section{Primary Author:}

Benjamin P. S. Donitz, Jet Propulsion Laboratory, California Institute of Technology

Contact information: benjamin.p.donitz@jpl.nasa.gov,626-318-4029

\section{Co-Authors:}

Karen J. Meech, University of Hawai'i, Institute for Astronomy, meech@hawaii.edu Julie Castillo-Rogez, Jet Propulsion Laboratory, California Institute of Technology, julie.c.castillo@jpl.nasa.gov

Kimberly M. Moore, California Institute of Technology, kmmoore@caltech.edu Samuel W. Courville, Planetary Science Institute, Arizona State University, swcourville@psi.edu Sierra Ferguson, Arizona State University, Sierra.Ferguson@asu.edu Kristie LLera, Soutwest Research Institute, San Antonio, kristie.llera@swri.org Tibor Balint, Jet Propulsion Laboratory, California Institute of Technology, tibor.balint@jpl.nasa.gov

\section{Endorsers:}

Stefano Nerozzi (Lunar and Planetary Laboratory, University of Arizona), Ariel N. Deutsch (Brown University), Jesse Tarnas (Brown University), Jeffrey Stuart (Jet Propulsion Laboratory, California Institute of Technology), Ellen C. Czaplinski (University of Arkansas), Donald H. Kuettel III (University of Colorado, Boulder), Alexander E. Thelen (NASA Goddard Space Flight Center; Universities Space Research Association), Ashley Schoenfeld (University of California, Los Angeles), George Clark (Johns Hopkins University Applied Physics Laboratory), Cyril Simon Wedlund (Space Research Institute, Austrian Academy of Sciences, Graz, Austria), Dogacan Su Ozturk (Jet Propulsion Laboratory, California Institute of Technology), Jim Burch (Southwest Research Institute), Stephen Fuselier (Southwest Research Institute), Paul Tenfjord (Space Plasma Physics Group, University of Bergen, Norway), Peter Buhler (Jet Propulsion Laboratory, California Institute of Technology) 


\section{Introduction}

Oort Cloud Comets (OCCs) are fossil records of the origin of the Solar System. Occasionally, a passing star or rogue planet disrupts the tenuous bond that ties an OCC to its faraway orbit in the Oort Cloud, sending it on a sometimes million year journey towards the inner solar system. C/1995 O1 (Hale-Bopp) was the last Great OCC to pass through the inner solar system, capturing the world's attention as it streaked through the night sky with a brightness rivaled only by Sirius and a dust tail that at times extended 45 degrees. What was more challenging to observe from Earth, however, were the volatiles that had been sealed in the icy regimes of the Oort Cloud since the formation of the solar system. Though astronomers on Earth were able to make significant observations of comet Hale-Bopp in a variety of spectra using both Earth-based assets and existing space-based assets (like Ulysses), the quality of science could not rival that of close observations from a dedicated spacecraft.

We propose to develop mission concepts to encounter a pristine OCC, with near-surface ices preserved from the solar system's formation and primordial isotopic markers. Hence, OCC exploration has potential to address many key questions identified in the Planetary Science Decadal Survey (Vision and Voyages)(National Research Council, 2011), from understanding the initial stages of planet formation, to the origin of water on Earth, and planet migration and thus being responsive to NASA's strategic goal to understand "How did our solar system form and evolve?" Specific questions targeted by this concept include:

Q1. How did the solar system form from the protoplanetary cloud?

Q2. What is the history of solar system volatile and organic compounds?

Q3. What was the role of comets in the delivery of water to the inner solar system?

Q4. How can measured comet chemistry be related to formation location or evolutionary processing history? What roles do evolutionary processes play?

Q5. What is the detailed physical structure of comets, and how does this relate to the mechanisms of cometary activity?

Highlighting the exploration of OCCs - one of the last unexplored classes of solar system objectsin the next decadal survey's New Frontiers program is the natural next step in the long-term roadmap for deciphering the solar system origins contained in comets. It is the logical and necessary mission after the recent exploration of 67P/Churyumov-Gerasimenko by the Rosetta mission.

Though targeted exploration of OCCs has an extremely compelling science case, the challenges posed by the nature of these foundational targets make a mission to encounter one complex. The greatest challenges to exploring OCCs are their unpredictable orbits and detecting them far enough in advance to reach them before they depart to the edge of the solar system again. The planetary science and mission concept community is accustomed to conducting missions to periodic objects that have existed predictably for many millennia and will continue to persist for many more. The programmatic process of funding missions is well attuned to this paradigm and does not leave any room for responding to a target of opportunity. An OCC does not fit within this classical paradigm because its first observation is no less than 10 years before it reaches perihelion, and its long orbital 
period leaves no possibility of returning again for many thousands or millions of years. However, with novel mission design, encountering an OCC is likely feasible.

\section{We request that the Decadal Committee recommends funding of a mission concept study that would inform the mission portfolio for the 2023-2032 survey. \\ 2 Oort Cloud Comet Science}

Small primitive bodies preserve a record of processes that occurred as the solar system formed. To understand this record, we must measure the compositions of a range of primitive bodies from different locations in the solar system and compare them with the predictions from models of early solar system formation. Long period comets (LPCs) have been among the most difficult minor bodies to characterize due to their brief, "once-in-a-lifetime" passages through the inner solar system. Although LPCs are typically brighter than short period comets because their volatile inventory has not been depleted by repeated perihelia, their high activity levels also makes it difficult to characterize their nuclei, and LPCs are rarely discovered before they become active. In particular, LPCs that originate in the Oort Cloud, also known as Oort Cloud comets (OCCs), are mostly pristine since they have not been repeatedly exposed to high solar insolation. Encountering a pristine OCC that has near-surface ices and isotopic markers preserved from the solar system's formation would address many key questions identified in the previous Planetary Science Decadal Survey (Vision and Voyages). A dedicated White Paper submitted to the Decadal Committee, Mission Design Study for Objects on Oort Cloud Comet Orbiters by Meech and Castillo-Rogez, dives deeper into the science case for an LPC or OCC mission.

\section{Mission Concept Study Motivation}

Fully realizing the foundational science potential of OCCs will require a dedicated spacecraft mission. Although current telescopic assets such as Pan-STARRS and Hubble routinely observe long period comets, they have limited resolution and instrumentation. Additional follow-up observation time is also extremely competitive and hard to secure. Even next-generation telescopic assets like the Vera C. Rubin Observatory (LSST) or the James Webb Space Telescope (JWST) will have limited capability to observe OCCs. Thus, a dedicated mission is required to expand knowledge of these bodies. Furthermore, spacecraft observations can provide density constraints and reveal the surface topography of these mysterious objects with an order of magnitude higher resolution than ground-based observations. Spacecraft exploration would also allow for direct in-situ measurements of an OCC, such as with a mass spectrometer or an impactor experiment. Even when high cometary activity precludes in-situ observation or topology charterization, remote sensing opportunities can yield foundational science.

Recent advances in Earth-based telescopic assets over the past decade have significantly increased the detection rate of OCCs, making now an opportune time to formulate a mission concept to visit an OCC up close and acquire in-situ and high-resolution remote sensing measurements. Since 2010, the Pan-STARRS survey (Kaiser et al., 2002) has performed a systematic survey of the entire Hawaiian sky, allowing for the advanced detection of OCCs such as comets C/2011 L4 (Micheli et al., 2011) and C/2017 K2 (Meech et al., 2017); archive analysis revealed the latter comet was 
visible since 2013, about 10 years prior to perihelion. Next-generation telescopes will further increase the distance at which OCCs are detectable; the upcoming LSST has formally committed $90 \%$ of its telescope time to performing an all-sky survey. These facilities will strongly influence our catalog of solar system objects over the next decade and increase the feasibility of an OCC encounter.

Despite the important science value and increased detection rates of OCCs, only a few studies on their exploration have been performed thus far. One such study is Comet Interceptor, which is a European Space Agency mission that will launch to a stable parking orbit and await an OCC accessible within the $\Delta_{V}$ resources of that mission. Comet Interceptor has a maximum bus cost of $150 \mathrm{M}$ Euros ( $\sim$ \$175MFY20) (Snodgrass and Jones, 2019), making it similar to a Discovery class NASA mission. Another mission concept, Xenia (Donitz et al. 2020; Matousek et al. 2020), looked into a rapid flight system design and development to explore the dynamically new comet C/2017 K2, leveraging the methodology and lessons learned from the Mars CubeSat One (MarCO) CubeSats. While these existing concepts show that missions to OCCs are feasible, a follow-on activity can build on their returned science with a larger payload suite and more focused instruments. This emphasizes the need for a dedicated New Frontiers-class mission concept study.

\section{Scope of Mission Concept Study}

The goals of the new proposed study are (1) to design a science concept addressing the questions noted above; (2) to work with a study team at one of the design centers to define the most affordable architecture that would achieve these objectives with technologies available in the next decade; (3) to compare different observational strategies to address the science objectives, leading to a portfolio of concepts for the future planetary science decadal survey (DS) committee to assess when developing the 2023-2032 DS; (4) to identify technologies that require investment in the next decade in order to support this concept; and (5) to deliver that information in the form of a report to NASA's Planetary Science Division and in the form of presentations to the community.

The main mission architectural trade is to assess the relative strengths and weaknesses to either (1) a reactive mission, built on the ground in response to the discovery of a "Great" comet 5 to 10 years ahead of perihelion, or (2) a proactive mission, that is built, launched, and stationed on a standby orbit in near-Earth space (e.g., Earth-Moon L2) from which it could be launched to an inbound "Great" comet. The science team will develop a set of triggering conditions to determine what type of comet target is scientifically meritorious and accessible in a reasonable time-frame. A characterization of the phase space of targets accessible for a range of launch energies is illustrated in Fig. 1.

In both scenarios the spacecraft would encounter the comet as it crosses the ecliptic. As a reference, it takes about 6 months for a spacecraft to travel from Earth and meet a comet at $\sim 2 \mathrm{AU}$. The target may be observable during this entire period with the remote sensing payload: visible camera, UV spectrometer, NIR spectrometer. A few hours ahead of closest approach, sub-millimeter wave spectroscopy would be used to characterize the $\mathrm{D} / \mathrm{H}$ and possibly the oxygen isotopes. During closest approach, all instruments would be used. In order to guarantee science return under challenging and risky observing conditions, the architecture will explore the value of using a frac- 


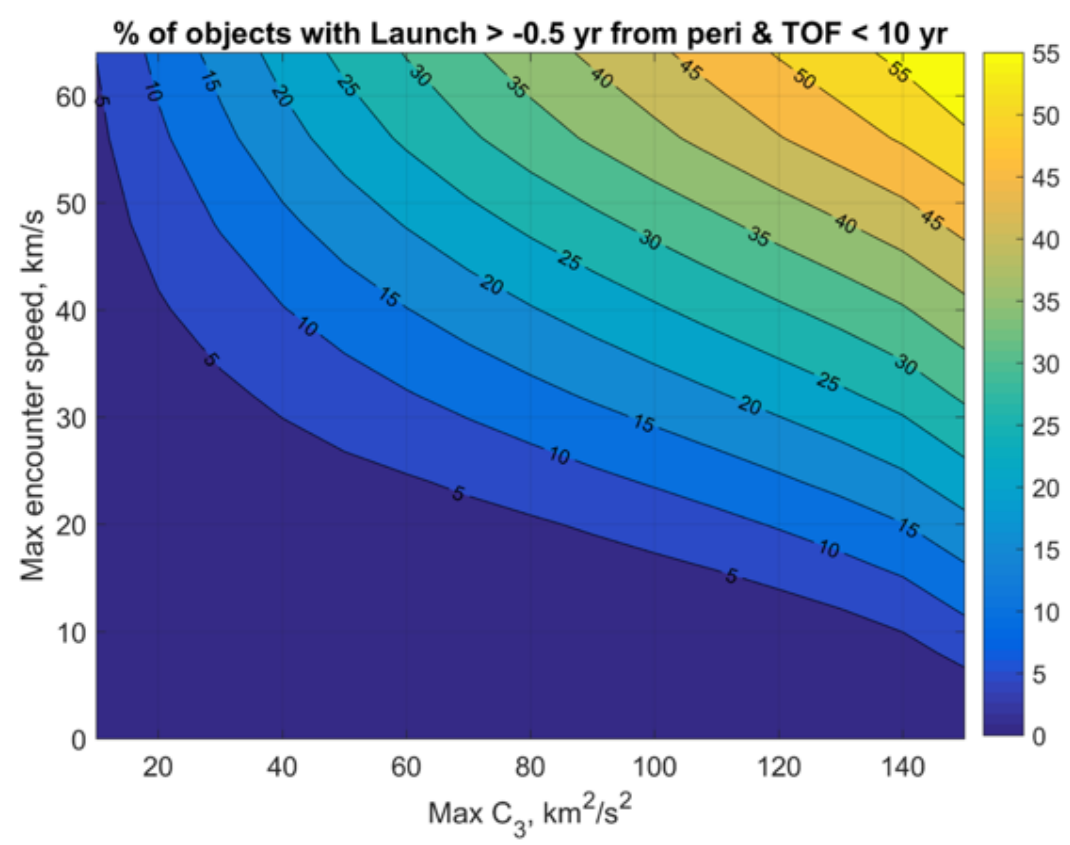

Figure 1: Probability of encountering comet accessible with $<10$ years $T O F$

tionated architecture involving a mothership and multiple smallsats deployed from the mothership. The concept will also leverage autonomous mission operations techniques that have been maturing over the past decades. These two novel aspects and a third ground-storage option are addressed in more detail below.

\section{Rapid Response Mission Architectures}

The most viable strategy for exploring an OCC is a "rapid response" mission architecture. This strategy enables the immediate development or launch of a spacecraft in response to a newly identified target, and employs rapid spacecraft development, implementation, and/or integration time. This flexibility of this strategy is ideal for visiting targets of opportunity such as OCCs, since these objects are only discoverable shortly before their perihelion, and will not return again in our lifetime.

Within the broad framework of a rapid response mission to explore an OCC, there are multiple possible approaches, each with their benefits and drawbacks. The main differences between the architectures relate to when the mission would complete key milestones (such as implementation and launch) relative to the discovery of a target object. Table 1 describes, at a high level, three identified architectures: building and storing a spacecraft on the ground until a target OCC is discovered, building and launching a spacecraft to a stable orbit while waiting for a target OCC to be discovered, or building and launching a spacecraft after an target OCC has been discovered.

The architectures also be assume different explicit risks to the project schedule, cost, technical implementation, or operations. Architectures that have accelerated implementation schedules inherit more schedule risk; architectures that require "standing armies" for indefinite periods of time in- 
herit greater cost risk; and architectures that require extended periods of dormancy inherit greater operations risk. Additional work is required to characterize, quantify and compare these risks across architectures.

Table 1: Rapid Response Mission Architecture Descriptions and Risks

\begin{tabular}{|c|c|c|}
\hline Architecture Description & Benefits & Drawbacks \\
\hline $\begin{array}{l}\text { [A1] - Build a spacecraft } \\
\text { and store it on the ground } \\
\text { until a compelling object ar- } \\
\text { rives }\end{array}$ & $\begin{array}{l}\text { - Reduces the risk of building } \\
\text { a spacecraft on a very aggressive } \\
\text { schedule compared to other archi- } \\
\text { tectures } \\
\text { - Allows for rapid launch after tar- } \\
\text { get discovery, since the spacecraft is } \\
\text { already built }\end{array}$ & $\begin{array}{l}\text { - Requires long-term storage which would have } \\
\text { adverse cost implications } \\
\text { - Requires a "standing army" of on-call engi- } \\
\text { neers, since a suitable target may be discovered } \\
\text { at any time during the storage window } \\
\text { - Requires flexible launch dates with a quick } \\
\text { launch turnaround time after target discovery, as } \\
\text { a launch slip could threaten mission failure } \\
\text { - No opportunity to upgrade to current state- } \\
\text { of-the-art spacecraft technology once a target is } \\
\text { identified }\end{array}$ \\
\hline $\begin{array}{l}\text { [A2] - Launch a spacecraft } \\
\text { to a stable orbit and wait un- } \\
\text { til a compelling object ar- } \\
\text { rives }\end{array}$ & $\begin{array}{l}\text { - The spacecraft can conduct addi- } \\
\text { tional science, such as astrophysics, } \\
\text { when dormant in orbit, though this } \\
\text { may require additional, potentially } \\
\text { costly instrumentation } \\
\text { - Minimizes risk of not encoun- } \\
\text { tering a target due to missing a } \\
\text { launch window, since the spacecraft } \\
\text { is launched in advance of target dis- } \\
\text { covery }\end{array}$ & $\begin{array}{l}\text { - Orbiting spacecraft needs to be maintained by } \\
\text { ground operators. This could be mitigated with } \\
\text { added autonomy } \\
\text { - Risks of faults or instrument degradation due to } \\
\text { the radiation environment in space } \\
\text {-Like case A1, the mission would also require a } \\
\text { "standing army" of engineers ready to assist with } \\
\text { the mission for an indefinite period of time }\end{array}$ \\
\hline $\begin{array}{l}\text { [A3] -Start building the } \\
\text { spacecraft only after an ob- } \\
\text { ject of interest has been } \\
\text { identified }\end{array}$ & $\begin{array}{l}\text {-The spacecraft is designed, built, } \\
\text { and outfitted with precisely what } \\
\text { would be needed for this particular } \\
\text { object } \\
\text {-Enables modifying the instrument } \\
\text { suite based on the specific target } \\
\text {-Enables most current technology }\end{array}$ & $\begin{array}{l}\text { - Highest schedule risk. It is possible that a } \\
\text { spacecraft could not be built in time for the en- } \\
\text { counter. } \\
\text { - Accelerated design-build-test process has in- } \\
\text { herent risk } \\
\text { - May reduce number of reachable targets com- } \\
\text { pared to A1 and A2, since the spacecraft cannot } \\
\text { launch immediately upon object discovery }\end{array}$ \\
\hline
\end{tabular}

Even with increased ability to detect OCCs 5-10 years out with new observatories like LSST, the current NF cadence still requires some form of rapid response. NF concept may take 8 years from development to launch, as demonstrated by OSIRIS-REx, which would require a nearly perfect alignment between OCC detection and AO release. This is not to say that the spacecraft must be entirely developed prior to $\mathrm{AO}$ release, but that this requires a novel approach to respond to targets of opportunity.

A rapid response and fast flyby mission requires advanced technologies in the payload and flight system architecture, many of which are currently at mature technology readiness levels (TRL). Multiple independent technologies currently being developed would also provide the capability required for a mission of this type. Remote spectroscopy instruments (ALICE, SwRI), organics mass spectrometers (QIT-MS, JPL), and dust spectrometers (SUDA, CU Boulder) that would enable 
high value science return are all at TRL levels greater than 5. Whipple shields, small deployables, and ob-board autonomous science processing (Thompson et al., 2012) that would technically enable an OCC encounter are also at TRL levels greater than 5. Synergistic developments in the field of autonomous science operations and fault management are quickly maturing and would increase the value of returned science and decrease overall mission risk.

\section{Conclusion}

A New Frontiers mission to encounter an OCC would deliver decadal-grade science, informing a generation of planetary scientists who study the formation of the solar system and diversity of comets. This first-of-its-kind mission would revolutionize the community's understanding of the nature of the edge of the protoplanetary disk. However, the modalities of this kind of mission remain to be established. The variance in architectures and the specific risks attributed to each creates a complex trade space to navigate for mission planners. A dedicated mission architecture study would address and resolve:

- The best approach to a rapid response mission architecture as a function of the target object's discovery time and orbital parameters

- Development of a flight system that enable investigations in proximity of a very active object.

- Further technology development recommendations in support of a mission of this class.

- Programmatic recommendations to enable a rapid response OCC mission. A separate white paper in development for the September 15 deadline will provide specific recommendations on policy changes that would allow for a rapid response mission.

We request that the Decadal Committee recommends funding of a mission concept study that would inform the mission portfolio for the 2023-2032 survey.

Ground breaking science can be accomplished at an OCC, filling an important gap in the understanding of the formation and evolution of the solar system. We hope the Decadal Committee considers the motivations and requests outlined in this white paper, and that the Decadal Survey advocate for a paradigm shifting mission in planetary science to visit an Oort Cloud Comet.

\section{Acknowledgements}

The research was carried out at the Jet Propulsion Laboratory, California Institute of Technology, under a contract with the National Aeronautics and Space Administration (80NM0018D0004). Pre-Decisional Information - For Planning and Discussion Purposes Only.

\section{References}

Donitz, B., Matousek, S., Castillo-Rogez, J., Balint, T., et al, M. R., 2020. Smallsat reactive flyby to oort cloud comets and interstellar objects. In: Interplanetary Small Satellite Conference, \& Pasadena, CA. 
Kaiser, N., Aussel, H., Burke, B. E., Boesgaard, H., Chambers, K., Chun, M. R., Heasley, J. N., Hodapp, K.-W., Hunt, B., Jedicke, R., Jewitt, D., Kudritzki, R., Luppino, G. A., Maberry, M., Magnier, E., Monet, D. G., Onaka, P. M., Pickles, A. J., Rhoads, P. H. H., Simon, T., Szalay, A., Szapudi, I., Tholen, D. J., Tonry, J. L., Waterson, M., Wick, J., 2002. Pan-STARRS: A Large Synoptic Survey Telescope Array. In: Tyson, J. A., Wolff, S. (Eds.), procspie, Volume 4836 of Society of Photo-Optical Instrumentation Engineers (SPIE) Conference Series, pp. 154-164.

Matousek, S. E., Castillo-Rogez, J. C., Donitz, B. P., Balint, T., 2020. Close Flyby and Characterization of Long Period Comets Like C/2017 K2. In: Lunar and Planetary Science Conference, Lunar and Planetary Science Conference, pp. 2831.

Meech, K. J., Kleyna, J. T., Hainaut, O., Micheli, M., Bauer, J., Denneau, L., Keane, J. V., Stephens, H., Jedicke, R., Wainscoat, R., Weryk, R., Flewelling, H., Schunová-Lilly, E., Magnier, E., Chambers, K. C., 2017. CO-driven Activity in Comet C/2017 K2 (PANSTARRS). ApJ 849, L8.

Micheli, M., Wells, L., Holmes, R., Foglia, S., Vorobjov, T., Sostero, G., Guido, E., Sato, H., Williams, G. V., 2011. Comet C/2011 L4 (Panstarrs). IAU Circulars 9215, 1.

National Research Council, 2011. Vision and Voyages for Planetary Science in the Decade 20132022. The National Academies Press, Washington, DC.

Snodgrass, C., Jones, G. H., 2019. The European Space Agency's Comet Interceptor lies in wait. Nature Communications 10, 5418.

Thompson, D., Castillo-Rogez, J., Chien, S., Doyle, R., Estlin, T., McLaren, D., 2012. Agile science operations: a new approach for primitive bodies exploration. In: SpaceOps 2012 Conference. 